\title{
518 Measurement of Impulsive Forces and Crater Formation in Impulse Discharge
}

\author{
Y.Kobayashi and T.Tamura* \\ *Department of Mechanical and Production Engineering, Niigata University, Niigata, Japan
}

\section{Summary}

Recently, many new machining technologies which utilize electrical discharge machining (EDM) have been developed. However, the mechanism of material removal in EDM has not yet been sufficiently explained. In this study, to clarify the mechanism of material removal, first, the transitions in the impulsive forces in an impulse discharge were measured. Second, how the impulsive forces affect the formation of a crater by impulse discharge was examined. The following results were obtained. (1) A typical waveform obtained from an impulse discharge in kerosene consists of primary, secondary, tertiary and quaternary waves, and the intervals between the peaks of waves gradually become smaller. (2) The peak value of the primary wave, ranging from 15 to $20 \mathrm{~N}$, depends on the peak current. The interval ranging from 200 to $350 \mu \mathrm{s}$ from the start of discharge to the peak of the secondary wave depends on the discharge energy. (3) Because the shape of the crater is strongly dependent on the polarities under the same working conditions in EDM, the effect of the impulsive force acting between the workpiece and the electrode on the formation of a crater by impulse discharge may be insignificant.

Key words: EDM, mechanism of material removal, impulsive forces, crater

\section{Introduction}

Electrical Discharge Machining (EDM) is widely used for manufacturing various kinds of dies and molds. But EDM has numerous problems such as low material removal rate (MRR) and the generation of heat affected zones (HAZs), in which there are numerous cracks and microcraters. However, many new machining technologies which utilize EDM have been developed recently. For example, micro-EDM technology ${ }^{(1)}$ using a simple-shaped electrode such as a cylindrical one has been developed. It realizes high-precision and complex-shape machining. Moreover, surface modification technology ${ }^{(2)}$ utilizing EDM that uses working oil mixed with silicon powder has been made practicable. However, the mechanism of material removal in EDM has not been sufficiently clarified. Its clarification is necessary for not only the understanding of the phenomena of EDM but also the development of new EDM technology which will alleviate problems such as low MRR and the generation of HAZs. For its clarification, the transitions in the melted and vaporized regions and the transitions in the impulsive forces that act on the melted region should be precisely examined to clarify the mechanism of material removal. Additionally, we must make a connection between them when we investigate the mechanism of material removal.

From this point of view, many experiments ${ }^{(3)}$ for measuring the impulsive forces had been carried out as the basic study of EDM. However, in some experiments for measuring the impulsive forces conducted recently, results ${ }^{(4)(5)}$ different from the conventional ones have been reported.

In this study, to clarify the mechanism of material removal in EDM, first, the impulsive forces in an impulse discharge were measured. Second, by means of the observation of a crater, the mechanism of crater formation by impulse discharge was examined.

\section{Experimental method}

2.1 Experimental device for measuring the impulsive forces

Figure 1 shows the schematic illustration of the experimental device used for measuring the impulsive forces. The load cell consisted of piezoelectric element $\mathrm{SiO}_{2}$ whose natural frequency is $300 \mathrm{kHz}$ as the sensor for detecting the impulsive forces (PCB PIEZOTRONICS, $209 \mathrm{C} 1$ ). The workpiece was made of $18 \mathrm{Cr}-8 \mathrm{Ni}$ stainless steel (JIS; SUS304). It was formed to have a cylindrical shape with a $4 \mathrm{~mm}$ diameter and $0.2 \mathrm{~mm}$ thickness, and was bonded to the sensor with bonding material. To prevent attenuation of the impulsive forces, the bonding material was very thin. The measurement system

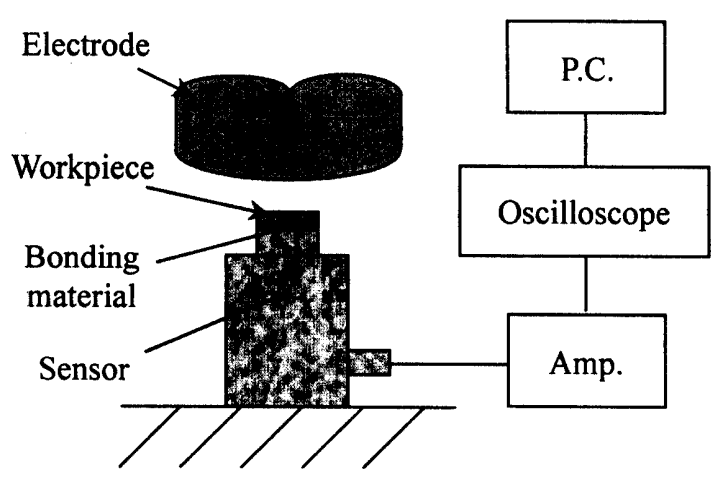

Fig.1 Experimental device for measuring the impulsive forces 
consisting of a sensor, workpiece, and bonding material constituted a mass-spring-damper system, and its natural frequency became $290 \mathrm{kHz}$. The signal outputted from the sensor by the action of impulsive forces was input to a digital storage oscilloscope (IWATSU, DS-8607) through an amplifier (PCB PIEZOTRONICS, 480E09) and was recorded on a personal computer.

2.2 EDM conditions

The electrode is cylindrical with an $18 \mathrm{~mm}$ diameter, and the radius of curvature of the tip is $70 \mathrm{~mm}$. The EDM conditions in this experiment are listed in Table 1. The working oil is kerosene which is the stationary state. Polarity is mainly reversed; the electrode is the anode and the workpiece is the cathode. The open circuit voltage is about $300 \mathrm{~V}$, gap voltage is about $17 \mathrm{~V}$ and gap length is about $200 \mu \mathrm{m}$.

Table 1 EDM conditions

\begin{tabular}{c|c}
\hline Peak current $i_{\mathrm{e}}[\mathrm{A}]$ & $3.3 \sim 13.2$ \\
\hline Pulse duration $t_{\mathrm{e}}[\mu \mathrm{s}]$ & $2 \sim 80$ \\
\hline Polarity & reversed and straight \\
\hline Dielectric fluid & kerosene \\
\hline
\end{tabular}

3. Results and discussion

3.1 Impulsive forces generated by impulse discharge in kerosene

Figure 2 shows a typical waveform obtained from an impulse discharge in kerosene under the condition that peak current is $13.2 \mathrm{~A}$, pulse duration is $60 \mu \mathrm{s}$ and polarity is reversed. As shown in Fig.2, the waveform consists of primary(1), secondary(2), and tertiary(3) waves, and gradually attenuates with the passage of time.

It is necessary to verify whether the measurement system in our experiment precisely detected the impulsive forces or not. In our experiment, the validity of the waveform of impulsive forces obtained from an impulse discharge is evaluated with respect to $f_{e} / f_{n}$ (the ratio of the frequency of impulsive forces $f_{e}$

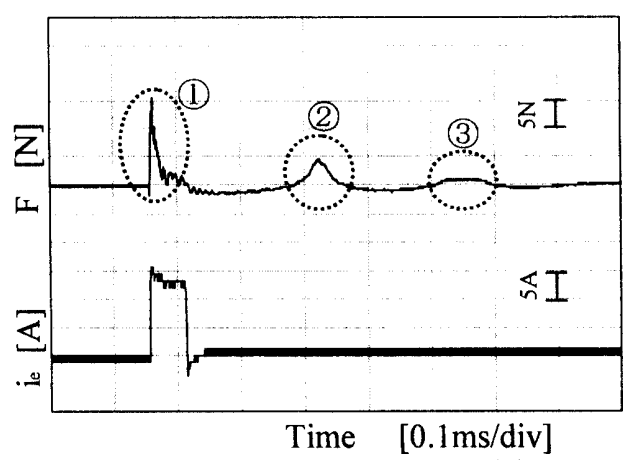

Fig.2 Typical waveform obtained from an impulse discharge in kerosene to the natural frequency of the measurement system $f_{n}$ ). When the waveform of impulsive forces consisting of various frequency components is analyzed, we need to obtain the highest frequency component of all impulsive forces. From this point of view, as the result of FFT analysis, the frequency at the beginning of the primary wave was the highest at $103 \mathrm{kHz}$. As the result, $f_{e} / f_{n}(0.36)$ was very small. Thus, it is expected that the measurement system in our experiment precisely detects the impulsive forces. The time constant of the measurement system was not taken into consideration in this experiment.

3.2 Impulsive forces generated by impulse discharge in air

Figure 3 shows a typical waveform obtained from an impulse discharge in air under the condition that peak current is $13.2 \mathrm{~A}$, pulse duration is $60 \mu \mathrm{s}$ and polarity is reversed. Clearly, Fig. 3 is different from Fig.2; the flat waveform was obtained in air. This means that the impulsive forces in air could not be detected under the experimental conditions (e.g., detection sensitivity of the sensor) similar to those for kerosene. Therefore, electrical forces such as electromagnetic force are considered to be very small. Thus, electrical forces are not the primary influencing factor in Fig.2. From this, we inferred once again that working oil plays a very important role in the generation of the impulsive forces shown in Fig.2. In other words, it is expected that a primary factor responsible for the impulsive forces obtained in the experiment in kerosene is the force due

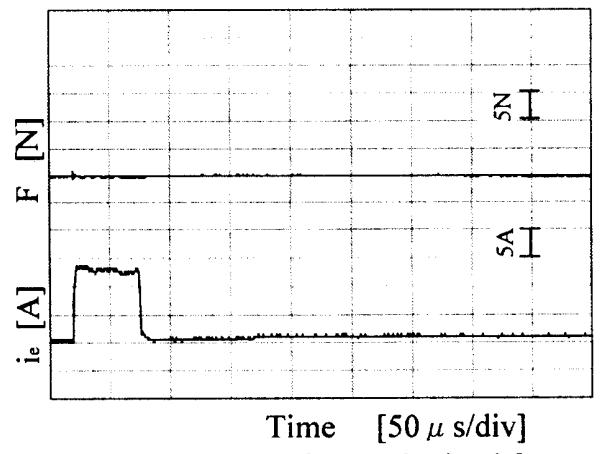

Fig.3 Typical waveforms obtained from an impulse discharge in air 
to expansion and shrinkage motions of a bubble.

3.3 Relationship between EDM conditions and the impulsive forces

To examine the effect of EDM conditions on the impulsive forces, experiments using the device shown in Fig. 1 for measuring the impulsive force were carried out under the conditions listed in Table 1 . Here, the peak of the primary wave $\left(F_{1}\right)$ and the interval from the primary wave to secondary wave $\left(t_{12}\right)$ were observed and were examined with changing the EDM conditions. The reason why $F_{1}$ and $t_{12}$ were selected is as follows.

When analyzing the formation mechanism of a crater generated by impulse discharge, it is expected that the region melted by impulse discharge is effectively removed by the primary wave and by the secondary wave if it acts during the melting of the workpiece. In other words, it is expected that these play important roles in the process of material removal. Thus, these two factors $\left(F_{1}\right.$ and $\left.t_{12}\right)$ were selected.

3.4 Effect of EDM conditions on $F_{1}$

First, the effect of peak current on $F_{1}$ was examined, where the pulse duration was kept constant and peak currents ranging from $3.3 \mathrm{~A}$ to $13.2 \mathrm{~A}$ were used. $F_{1}$ was examined from waveforms obtained from the experiments, and the results are shown in Fig.4. Second, the effect of pulse duration on $F_{1}$ was examined, where the peak current was kept constant and pulse durations ranging from $10 \mu$ s to $80 \mu \mathrm{s}$ were used. The results are shown in Fig.5. As shown in Fig.4, when the peak current increased from 3.3A to $13.2 \mathrm{~A}, F_{1}$ showed an increase of about $5 \mathrm{~N}$, from $15 \mathrm{~N}$ to $20 \mathrm{~N}$. But as shown in Fig. $5, F_{1}$ showed little change even if the pulse duration was increased from $10 \mu \mathrm{s}$ to $80 \mu \mathrm{s}$. The reason for this may be as follows. It is expected that the primary factor responsible for the primary wave is the shock wave generated by a sudden increase of pressure of the arc column and the area around it. Therefore, the larger the peak current is, the higher the energy in the gap is. Therefore, the distribution of pressure immediately after the start of discharge is high. As a result, a large shock wave was generated and $F_{1}$ was also large. However, when the peak current is constant, the pressure distribution immediately after the start of discharge was constant regardless of the pulse duration. Namely, the pulse duration had no serious effect on $F_{1}$. Thus, it was found that $F_{1}$ was dependent on the peak current.

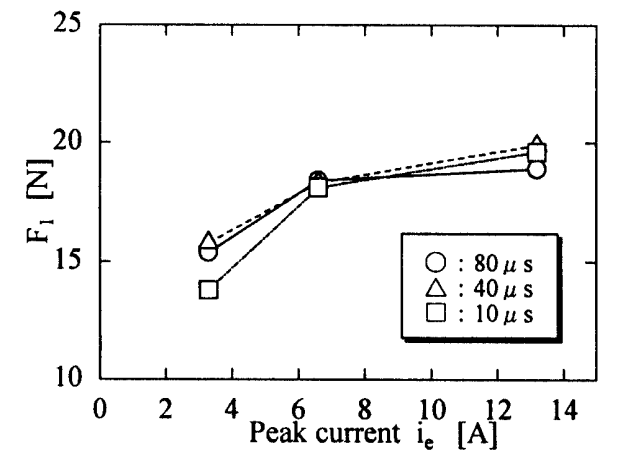

Fig.4 Relationship between peak current and $F_{1}$

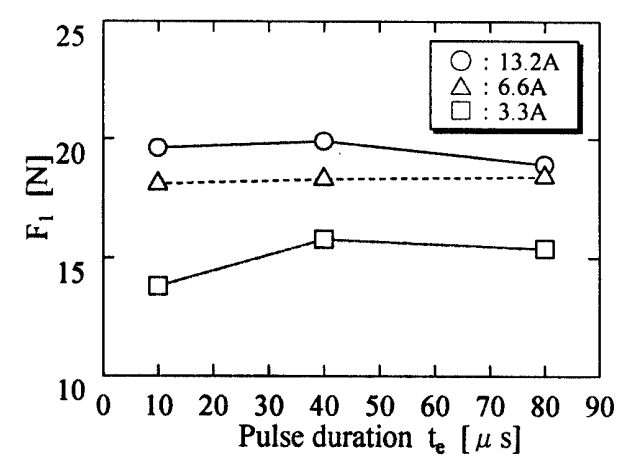

Fig.5 Relationship between pulse duration and $F_{1}$

\subsection{Effect of EDM conditions on $t_{12}$}

Figure 6 shows the relationship between $t_{12}$ and peak current under certain pulse durations, and Fig.7 shows the relationship between $t_{12}$ and pulse duration under certain peak currents. As shown in Fig.6 and Fig. $7, t_{12}$ ranges from $200 \mu \mathrm{s}$ to $350 \mu \mathrm{s}$ and shows a tendency to increase with increasing peak current and pulse duration. As a result, it is expected that $t_{12}$ is dependent on the discharge energy. Thus, from these results, the relationship between $t_{12}$ and discharge energy is shown in Fig.8. In Fig.8, $t_{12}$ shows a tendency to increase with the increase of discharge energy. Moreover, two data sets are respectively shown in the regions bounded by the broken lines. These are the results obtained from the experiments performed with changing the peak current and pulse duration under the same discharge energy. From these results, even if peak current and pulse duration are different, $t_{12}$ is approximately constant under certain discharge energies.

From these, it was inferred that $t_{12}$ is dependent on discharge energy. This reason for this may be as follows. It is expected that $t_{12}$ is dependent on the motions of a bubble. Therefore, because an internal energy of a bubble increases with the increase of discharge energy, the period of motions of a bubble is long, namely, $t_{12}$ is also long.

Moreover, as shown in Fig.8, $t_{12}$ is close to $350 \mu \mathrm{s}$ with the increase of discharge energy. From this, in the case of pulse duration over $350 \mu$ s, it is suggested that a secondary wave acts during the discharge. This was confirmed in the experiment for the case of pulse duration over $350 \mu \mathrm{s}$. From the results mentioned above, it was found that $F_{1}$ is dependent on peak current, and that $t_{12}$ is dependent on the discharge energy and is close 


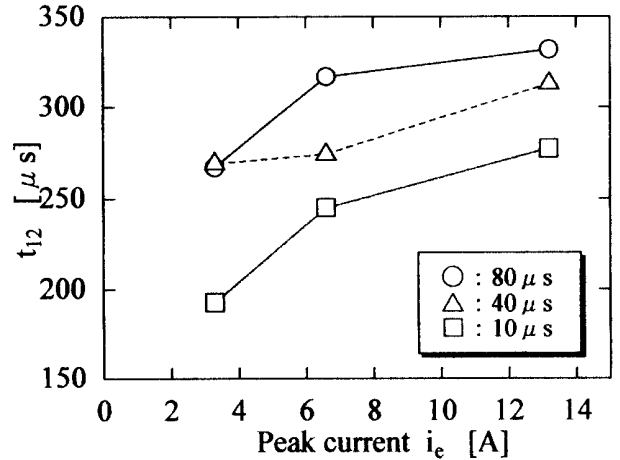

Fig.6 Relationship between peak current and $t_{12}$

\subsection{Crater generated by impulse discharge in kerosene}

Figure 9(a) shows a scanning electron microscope (SEM) image of a typical crater generated by impulse discharge under the condition that the peak current is $13.2 \mathrm{~A}$, pulse duration is 60 $\mu$ s and the polarity is reversed, and Fig.9(b) shows a profile along the broken line A-B in Fig.9(a). Under these conditions, the SEM image shows that the region melted by electrical discharge is effectively removed and a typical crater is formed. Moreover, because the crater is about $200 \mu \mathrm{m}$ in diameter and $15 \mu \mathrm{m}$ in depth as seen from Fig.9(b), it is considered that a bumpy crater is formed.

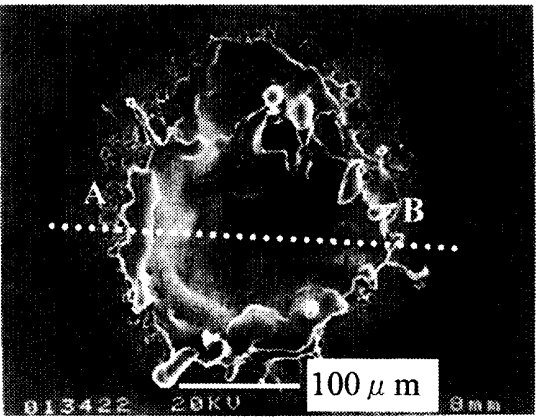

(a) SEM image

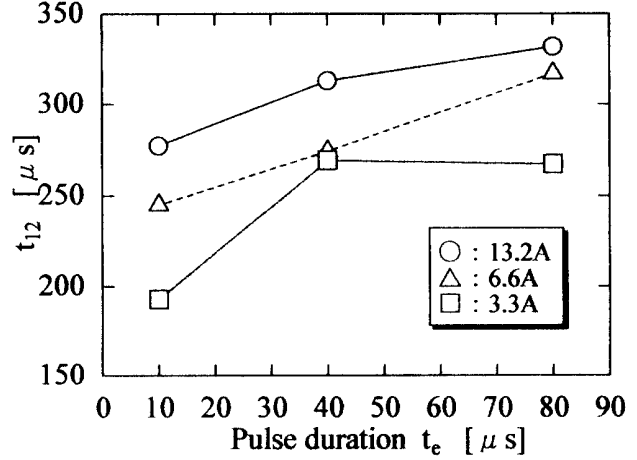

Fig.7 Relationship between pulse duration and $t_{12}$

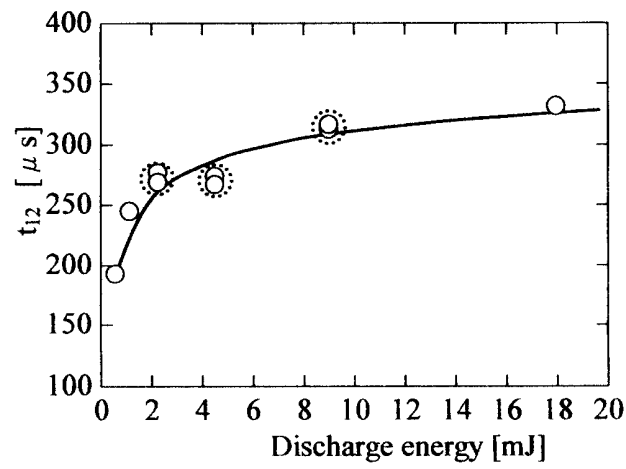

Fig. 8 Relationship between discharge energy and $t_{12}$

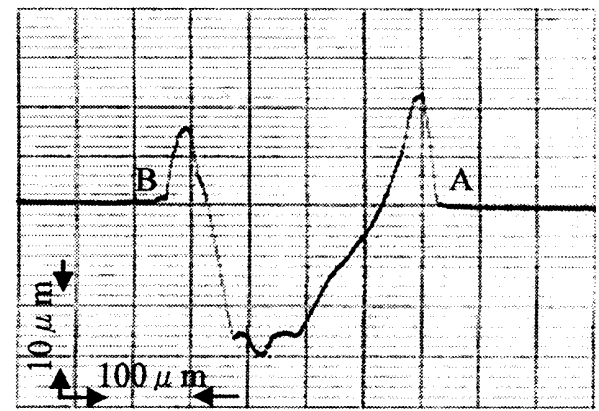

(b) Profile along A-B

Fig. 9 Crater generated by impulse discharge $\left(i_{\mathrm{e}}=13.2 \mathrm{~A}, t_{\mathrm{e}}=60 \mu \mathrm{s}\right)$

\subsection{Effect of the force caused by the motions of a bubble on crater formation}

To investigate the mechanism of crater formation by impulse discharge, on the basis of the results obtained from a series of experiments and the observation of craters generated by impulse discharge, the mechanism of crater formation in impulse discharge was examined.

The force due to the motions of a bubble generated in the gap has so far been regarded as the primary factor responsible for formation of a crater. From this, it is estimated that the crater shown in Fig.9(a) was formed by impulsive forces as shown in Fig.2. Judging from Fig.9(a) and (b), it is expected that some positive pressure acts on the melting region and the crater was formed by the removal of the melting region. Therefore, it is expected that the primary and secondary waves mainly affect the removal of the melting region.

However, a noteworthy phenomenon about the effect of force caused by motions of a bubble on crater formation was found. It is the effect of the polarity on crater formation.

To examine the effect of the polarity on crater formation, experiments with changed polarity, namely, experiments with straight polarity; the electrode is the cathode and the workpiece is the anode, were carried out. Figure 10(a) shows a typical waveform obtained from an impulse discharge in kerosene in the case of 


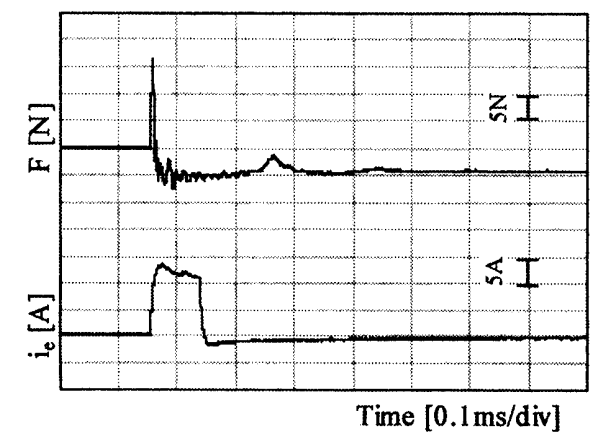

(a) Typical waveform

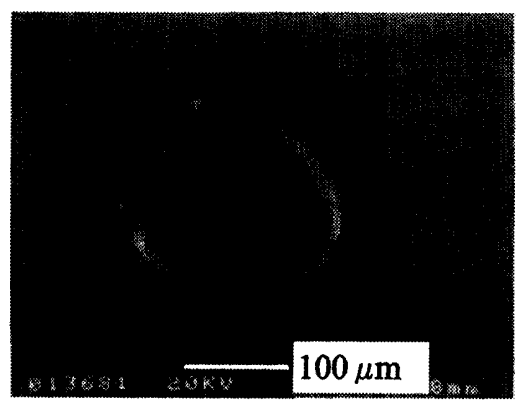

(b) SEM image

Fig.10 Typical waveform crater obtained from an impulse discharge in the case of straight polarity $\left(i_{\mathrm{e}}=13.2 \mathrm{~A}, t_{\mathrm{e}}=60 \mu \mathrm{s}\right)$

straight polarity.

As shown in Fig.10(a), the waveform of the impulsive force was similar to that in Fig.2 in the case of reversed polarity. Namely, Fig.10(a) appears to show a force caused by expansion and shrinkage motions of a bubble generated in the gap by impulse discharge.

Figure 10(b) shows a SEM image of the crater generated by impulse discharge in kerosene in the case of straight polarity. Figure 10(b) shows it to be different from the crater shown in Fig.9(a), namely, the former has a flat shape similar to the region melted by the discharge and then solidified without removal.

Moreover, to examine the effects of the polarity, the craters generated by impulse discharge in the case of reversed and straight polarities were observed. Fig.11(a) shows the craters generated by impulse discharge in
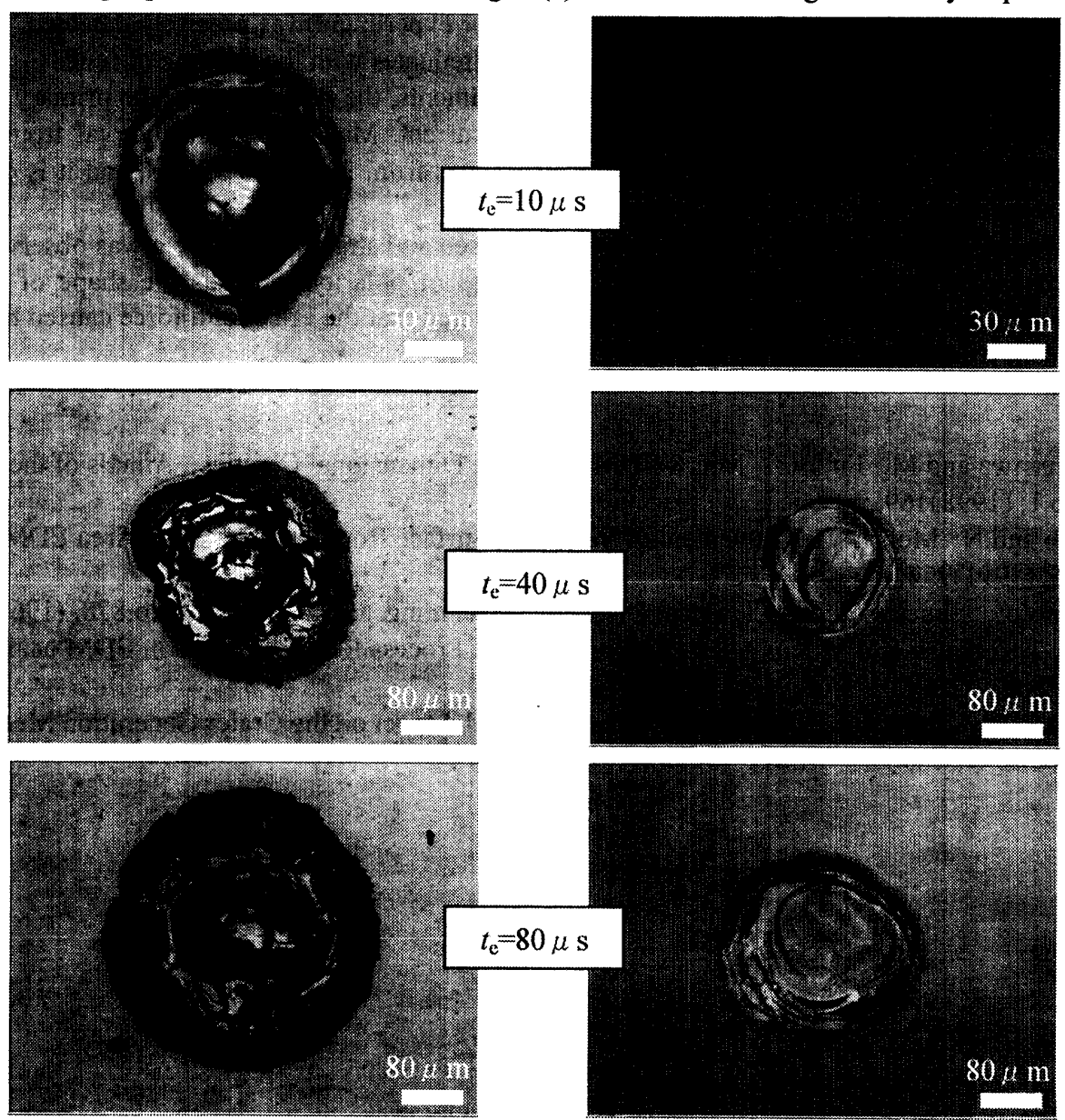

(a) Reversed polarity

(b) Straight polarity

Fig. 11 Typical craters generated by impulse discharge in kerosene $\left(i_{\mathrm{e}}=13.2 \mathrm{~A}\right)$ 
kerosene, under the condition that peak current is $13.2 \mathrm{~A}$, pulse durations are 10,40 and $80 \mu \mathrm{s}$, in the case of reversed polarity, and Fig.11(b) shows those in the case of straight polarity. As shown in Fig.11(a), bumpy craters were formed in reversed polarity. Meanwhile, as shown in Fig.11(b), flat craters were formed in straight polarity.

From these results, although a force caused by the motions of a bubble generated in the gap acted equally on the surface of the workpiece regardless of polarity, the shape of the crater was strongly dependent on the polarity. If the effect of the force caused by motions of a bubble on crater formation is significant, the shape of the crater in the case of straight polarity must be similar to that in the case of reversed polarity. That is to say, it appears that the effects of the force caused by motions of a bubble on crater formation are also insignificant. From this, craters in the case of reversed polarity, as shown in Fig.9 and Fig.11(a), were also found to be formed not under the effects of the force caused by motions of a bubble but under other factors.

As mentioned above, although we have so far expected that the force caused by motions of a bubble strongly affects crater formation, it appears that its effect on crater formation is insignificant. In short, at present, we can estimate that the effect of the force caused by motions of a bubble that has been regarded as the primary factor responsible for formation of a crater on the removal of the melting region is insignificant. However, as shown in Fig.9 and Fig.11(a), it is evident that the melting region was removed by some positive pressure. In future, it is necessary to further examine how a crater is formed.

\section{Conclusions}

To clarify the mechanism of material removal in EDM, impulsive forces generated by impulse discharge were measured using a measurement system, which has a natural frequency of $290 \mathrm{kHz}$, to detect the impulsive forces precisely. Moreover, craters formed under impulse discharge were observed and the mechanism of crater formation was examined.

(1) The impulsive force caused by the expansion and shrinkage motions of a bubble generated in the gap by impulse discharge using the measurement system in our experiment was precisely detected. It consists of primary, secondary and tertiary waves, and gradually attenuates with the passage of time.

(2) In the case of the EDM conditions used in these experiments, the peak value of the primary wave ranges from $15 \mathrm{~N}$ to $20 \mathrm{~N}$, and it is dependent on the peak current. Meanwhile, the interval from the start of discharge to the generation of the secondary wave ranges from $200 \mu \mathrm{s}$ to $350 \mu \mathrm{s}$, and it is dependent on the discharge energy.

(3) From the experiments performed for the cases of reversed and straight polarity, it was observed that force caused by motions of a bubble is the same regardless of polarity, whereas the shape of a crater was strongly dependent on the polarity. Namely, it is considered that the effect of a force caused by motions of a bubble on crater formation is insignificant.

References

(1) T. Masuzawa and M. Fujino: Micro-EDM for Three Dimensional Cavities, Annals of the CIRP, Vol. 47, No.1, (1998)169.

(2) N. Saito and N. Mohri: Improvement of Machined Surface Roughness in Large Area EDM, JSPE, Vol. 57,No.6, (1991)954.

(3) Y. Kimoto: Shock Force in Electrical Discharge Machining, JIEEJ, Vol.81, No.878, (1961)1877.

(4) M.Tohi, T.Komatsu and M.Kunieda: Measurement of Process Reaction Force in EDM using Hopkinson Bar Method, JSPE, Vol.68, No.6, (2002)822.

(5) Y. Uno, O. Endo and T. Nakajima: Fundamental Aspect od the Crater Generation Mechanism by a Single Pulse Discharge, JSEME, Vol.25,No.49, (1991)9. 